\title{
Negative effects of wind speed on individual foraging performance and breeding success in little penguins
}

\author{
Claire Saraux, $, 1,6$ André Chiaradia, ${ }^{2}$ Marcus Salton, ${ }^{2}$ Peter Dann, ${ }^{2}$ and Vincent A. Viblanc $3,4,5$ \\ ${ }^{1}$ Institut Français de Recherche pour l'Exploitation de la Mer (Ifremer), UMR MARBEC, Avenue Jean Monnet, CS 30171, \\ 34203 Sète cedex, France \\ ${ }^{2}$ Research Department, Phillip Island Nature Parks, PO Box 97, Cowes, VIC 3922 Victoria, Australia \\ ${ }^{3}$ Département Ecologie, Physiologie et Ethologie (DEPE), Institut Pluridisciplinaire Hubert Curien (IPHC), Université de \\ Strasbourg, 23 rue Becquerel, 67087 Strasbourg, France \\ ${ }^{4}$ CNRS, UMR7178, 67087 Strasbourg, France \\ ${ }^{5}$ Centre d'Ecologie Fonctionnelle et Evolutive, UMR 5175, CNRS-Université de Montpellier-Université Paul-Valéry \\ Montpellier-EPHE, 1919 route de Mende, 34293 Montpellier, France
}

\begin{abstract}
Most effects of environmental and climate variability on predator life history traits and population dynamics result from indirect effects mediated through the food chain. There is growing evidence that wind strength might affect seabirds while foraging at sea. Here, we investigated the effect of wind speed on the foraging performance of a flightless marine predator, the little penguin (Eudyptula minor). To this end, we used satellitederived wind data collected over 11 breeding seasons during which the daily attendance and body mass changes of more than 200 penguins breeding at Phillip Island (Victoria, Australia) were recorded by an automated penguin monitoring system. Over 17363 foraging trips, we found that wind speed had important effects on foraging and provisioning parameters in breeding adults. During incubation and chick-guard, stronger winds were associated with decreased foraging efficiency (lower body mass gain). During chick-guard, stronger winds were furthermore associated with lower meal sizes provided to the chicks, but parental body reserves appeared unaffected. Under extreme wind conditions $(>14 \mathrm{~m} / \mathrm{s})$ during the post-guard phase, adults maintained their body reserves by shifting towards longer foraging trips, while providing chicks with smaller meals. Chick meal size and foraging trip duration during chick rearing had direct effects on breeding success, suggesting that the influence of wind on individual fitness was mediated by changes in foraging performances and success. Furthermore, using a long-term wind data series spanning 150 -yr from a coastal wind station, we found a slight decline in wind speed and a decrease in wind speed variability in the Bass Strait where little penguins forage. Interestingly, based on this wind data, we found birds to be more directly affected by punctual events of strong winds (e.g. storms or gales), than by an overall change in wind patterns over time. Potential candidate mechanisms mediating the effects of wind speed on foraging efficiency may include swell formation, energy costs of travelling and thermoregulation, and a possible disruption of thermoclines, which may be important for little penguins. Plasticity in foraging strategies allowed parents to partially compensate for negative wind effects.
\end{abstract}

Key words: body condition; chick rearing; climate change vs. climate variability; Eudyptula minor; extreme events; seabird.

\section{INTRODUCTION}

A growing concern in ecology is the extent to which changes in climate affect species distribution, phenology, behavior, and life history (Kappelle et al. 1999, Hughes 2000, Stenseth et al. 2002, Lane et al. 2012, Plard et al. 2014). Much work has focused on determining the effects of changes in temperature or precipitation regimes on species and ecosystems functioning (Walther et al. 2002, Parmesan 2006). Such changes have been linked to modifications in bird, mammal, and insect phenol-

Manuscript received 10 November 2014; revised 6 August 2015; accepted 21 September 2015; final version received 14 October 2015. Corresponding Editor: J. P. Y. Arnould.

${ }^{6}$ E-mail: claire.saraux@gmail.com ogy (Parmesan 2006), including the time and duration of breeding (Zann et al. 1995, Forchhammer et al. 1998, Bale et al. 2002, Lane et al. 2012), affecting ecosystem assemblages and ecological networks (reviewed in Walther 2010). Studies have also considered how acclimatization and local adaptation may enable organisms to cope with increasing temperature trends linked to global warming (e.g. in sea coral species, Palumbi et al. 2014).

In this context, wind variability may be an important factor affecting animal behavior (Fairbanks and Dobson 2007, Elliott et al. 2014), physiology (Bize et al. 2010), or life history patterns (Weimerskirch et al. 2012). In seabirds, for instance, variability in wind speed may affect the energy costs of transportation during flight (Weimerskirch et al. 2000, Amélineau et al. 2014, Elliott et al. 2014) or 
foraging strategies (Dehnhard et al. 2013, Elliott et al. 2014, Lewis et al. 2015). Fewer studies have demonstrated the consequences of such variability on components of individual fitness (Kitaysky and Golubova 2000, Ganendran et al. 2011, Weimerskirch et al. 2012). However, studies investigating both ultimate effects of wind variability on fitness components and the proximate mechanisms through which those components are affected are scarce if not inexistent. Importantly, wind dynamics may incur indirect effects on animal fitness through ecosystemmediated processes (Ganendran et al. 2011).

In marine ecosystems, winds are prominent drivers of ecosystem productivity, affecting both the movement of nutrients through the ecosystem (via currents, waveswell) and the stratification of the water column (Klein and Coste 1984, Garrison 2011). Such effects can be measured in marine predator species, which typically integrate the effects of climate forcing throughout the relatively short oceanic food chains (Boyd and Murray 2001, Forcada et al. 2005, Trathan et al. 2007). For these species, climate effects on fitness are likely mediated by changes in availability and accessibility of prey and foraging success (Gjerdrum et al. 2003, Le Bœuf and Crocker 2005, Carol and Congdon 2007).

One way to test for such effects is to study fine-scale, short-term changes in the body mass of adult predators that can reliably be attributed to changes in adult body reserves and the size of meals provided to the offspring. We recently took this approach (see Saraux et al. 2011a) for little penguins (Eudyptula minor) foraging along the southern coast of Australia and New Zealand (Williams 1995). Little penguins are inshore foragers (Collins et al. 1999, Chiaradia et al. 2010) that visually hunt at sea during daytime (Cannell and Cullen 1998, Collins et al. 1999, RopertCoudert et al. 2006) and only return ashore after sunset (Klomp and Wooller 1991, Chiaradia and Nisbet 2006, Chiaradia et al. 2007). Using an Automatic Penguin Monitoring System (APMS; Kerry et al. 1993) that monitors changes in adults' body mass between foraging trips, we were able to determine the size of the meal fed to the chicks and highlight plasticity in little penguin foraging strategies to deal with the trade-off between self-maintenance and reproduction (Saraux et al. 2011a, Ramírez et al. 2015). We found that breeding adults alternated between short foraging trips $(\leq 2 \mathrm{~d})$ to feed their chicks and long trips ( $\geq 3 \mathrm{~d})$ to restore adult body reserves (Saraux et al. 2011a). Here, we build upon those findings to assess whether foraging success and individual fitness are affected by wind conditions throughout the breeding season.

An integrative understanding of how wind might affect little penguins in their marine ecosystem requires consideration of two important effects. The first is the extent to which these predators are affected by wind variability. For instance, variability in wind speed, direction, and the occurrence and frequency of extreme events may affect individual behavior, physiology, and reproduction (Weimerskirch et al. 2012, Elliott et al. 2014, Lewis et al. 2015), with consequences on population and ecosystem processes (see Parmesan et al. 2000 and references therein). The second is whether noticeable trends in wind variability occur over time. Animal susceptibility to changes in wind patterns over time would suggest that individuals are responding to climate change, and adaptations (or limits to adaptation) should then be considered (Visser 2008). In contrast, animals may respond to wind variability, but wind speed and/or direction remain stable over time. This would provide scope for investigations on how phenotypic plasticity might allow coping with variability and extreme events (Reed et al. 2011).

Here, we use data from 11 breeding seasons (20022012) of little penguins in southern Australia to investigate how variability in wind speed affects breeding success, potentially mediated by the proximate mechanism of disrupted foraging behavior, with effects on adult foraging success and chick provisioning (Saraux et al. $2011 a$ ). In addition, we investigated wind patterns over a 153-yr period (1861-2013) to identify whether noticeable changes in wind speed have been occurring in the fast changing climate of southeast Australia (Wu et al. 2012).

Little penguins provide a good model for investigating such questions. Off the southern coast of Australia, several penguin populations forage in the Bass Strait (Dann et al. 1992, Weavers 1992, Hoskins et al. 2008). High productivity is associated with shallow regions of the Bass Strait (Gibbs 1992, Afán et al. 2015), where a greater proportion of the water column is likely to mix under the action of wind. In addition, little penguins appear to rely to some extent on the stratification of water masses to locate their prey (Ropert-Coudert et al. 2009, Pelletier et al. 2012). When water masses are thermally stratified, prey location is apparently more efficient and foraging success higher than when water masses are thermally mixed, which seems to occur under strong wind regimes (Ropert-Coudert et al. 2009, Pelletier et al. 2012). Using data collected both at sea and from landbased stations, we explored whether wind speed in this region affected the foraging efficiency of breeding adults, namely the duration and efficiency (as measured by adult mass gain) of foraging trips during incubation and chickguard duties, and ultimately adult fitness.

\section{Methods}

\section{Long-term monitoring and field protocols}

We studied little penguins over 11 breeding seasons (2001/2002-2011/2012) in a colony of 28 000-32000 breeding adults (Sutherland and Dann 2012) on the western end of Phillip Island, Australia ( $\left.38^{\circ} 15^{\prime} \mathrm{S}, 143^{\circ} 30^{\prime} \mathrm{E}\right)$. The study site (see details in Chiaradia and Kerry 1999) contains over 100 artificial burrows (wooden nest boxes) of which 50-86 are occupied in each year. All nesting adults were first marked as chicks or when first found in the colony, with subcutaneous electronic transponders (TIRIS $^{\mathrm{TM}}$, Texas Instrument Registration and Identification System, Texas Instruments, Dallas, TX, USA and Allflex, Capalaba, Queensland, Australia) implanted 
under the skin between the scapulae. They were later sexed by bill measurements (Arnould et al. 2004) when first recaptured in the colony as adults in subsequent years. During the breeding season, nest boxes were checked three times a week. We only disturbed birds by opening the nest boxes for brief visual inspections when establishing laying, hatching, and fledging dates around the time of their expected occurrence (Chiaradia and Kerry 1999). For any other time, we used a handheld transponder reader capable of scanning transponder numbers through nest boxes to detect parents' presence with minimal bird disturbance (Chiaradia and Kerry 1999, Saraux et al. 2011a). Chick guard typically lasts for $2-3$ weeks and corresponds to the first stage of chick-rearing when chicks always have one parent present in the nest (Chiaradia and Kerry 1999, Chiaradia and Nisbet 2006). The post-guard phase corresponds to the subsequent period (up to fledging), when chicks are left alone in the colony and parents only briefly return to feed them. As little penguins usually lay two eggs (Williams 1995), the breeding success of penguin pairs was estimated as the number of fledged chicks compared to the number of eggs produced by the pair during the breeding season, using a binomial error distribution (see Statistics). In each year, the breeding success for the colony was quantified as the overall proportion of laid eggs resulting in fledglings.

\section{Foraging trips, adult body mass, and meal size}

Over the 11 breeding seasons, we recorded a total of 17363 foraging trips on 222 little penguins ( 110 males and 112 females). This corresponded to 2270 trips during incubation (yearly range $=132-296$ ), 3794 trips during chickguard (yearly range $=178-491$ ), and 11299 trips during post-guard (yearly range $=408-1447$ ). Foraging trips were recorded using the Automated Penguin Monitoring System (APMS) designed by the Australian Antarctic Division (Kerry et al. 1993) and located on one of the main colony entrances (Robinson et al. 2005). Penguins marked with transponders were automatically detected when walking over the APMS weighing platform on departure from, and arrival to, the colony. The APMS recorded the transponder number (individual ID), body mass (to the nearest $1 \mathrm{~g}$ ), and date and time of passage of each individual bird. As little penguins exclusively depart to sea before sunrise and return after sunset (Klomp and Wooller 1991), time of passage typically inferred arrivals between sunset and 1:00 a.m. local time or departures between 1:00 a.m. local time and sunrise (Chiaradia and Kerry 1999, Daniel et al. 2007). Foraging trip durations were calculated as the difference in days between bird movements in and out of the colony detected on the APMS platform (Chiaradia and Kerry 1999). Foraging trips were separated into short ( $\leq 2 \mathrm{~d}$ ) and long ( $\geq 3 \mathrm{~d}$ ) trips according to the distribution of trip durations (Saraux et al. 2011a). Both foraging trip duration (in days) and the proportion of short vs. long trips performed over the breeding season or over a breeding stage were used in subsequent analyses.
Adult body mass at each departure and arrival of a foraging trip was stored in the APMS and later retrieved for analysis. This enabled us to estimate three different mass parameters: (1) the body mass gained during a foraging trip; (2) the meal size given to the chicks; and (3) the change in adult body reserves (see Saraux et al. 2011a). Note that (2) and (3) are calculated only during chick rearing. Briefly, mass gain was defined as the change in mass during a foraging trip: mass gain (in grams) $=$ return body mass - departure body mass Chick meal size was the amount of food (in grams) given by an adult to its chicks and was estimated as the body mass lost by a bird between the time it entered and left the colony (Saraux et al. 2011a). Indeed, during chickrearing, adults only remain at the colony for a few hours to feed the chick (Daniel et al. 2007). Finally, adult reserve change corresponded to the body mass adults gained or lost after a foraging trip, once chick-meal mass was accounted for. It was calculated as the difference between adult body mass after the foraging trip once the chick was fed and the adult body mass before the trip. Raw data from the APMS were adjusted to account for tare drift and error of the system as described in Robinson et al. (2005).

\section{Wind data}

Wind speed and direction were obtained both from coastal weather stations (Bureau of MeteorologyBOM, Australia) and from satellite-derived data of the National Oceanic and Atmospheric Administration (NOAA). Coastal wind data came from Cape Otway wind station, situated in the foraging area of little penguins from this colony (Collins et al. 1999, Hoskins et al. 2008, McCutcheon et al. 2011, Pelletier et al. 2014, see map in Supplementary Materials, Fig. S1). We obtained a long-term time series (starting in 1861) from the Cape Otway weather station. As the number of per day measurements collected at this station varied over time, we worked on daily averages of only two measurements (9:00 and 15:00) that were continuously monitored over the entire period. This avoided spurious changes in data variance (Jakob 2010). This time series was used to investigate how wind patterns changed over time in the Bass Strait region. However, as data from this weather station were not recorded every day (due to logistic or technical problems), we used the satellite-derived sea-wind dataset provided by NOAA starting in 1987 (complete dataset for the entire 11 breeding seasons) to investigate wind effects on little penguins. Preliminary analyses showed that the wind direction and speed of the Cape Otway station and sea-wind dataset were highly correlated $(\rho=0.71, P<0.001, n=9381$ for wind speed). Over the 11 breeding seasons, daily wind speed varied from 3.8 to $18.6 \mathrm{~m} / \mathrm{s}$, the distribution showing a longer tail than expected in a normal distribution (Supplementary Materials, Fig. S1). Given that the effect of wind (e.g. on water mixing and thermoregulation) may 
be non-linear and operate above a certain threshold, wind speed was analyzed both as a continuous variable and as a two-level categorical variable: strong vs. usual winds. Strong winds (extreme events) were characterized as the tail events in the wind distribution, i.e. winds
$>14 \mathrm{~m} / \mathrm{s}$ (Supplementary Materials, Fig. S2), being representative of storm conditions (Dehnhard et al. 2013).

As little penguins are not synchronous breeders, the number of penguins breeding on any given day varies substantially. Thus, wind conditions on a given day may have
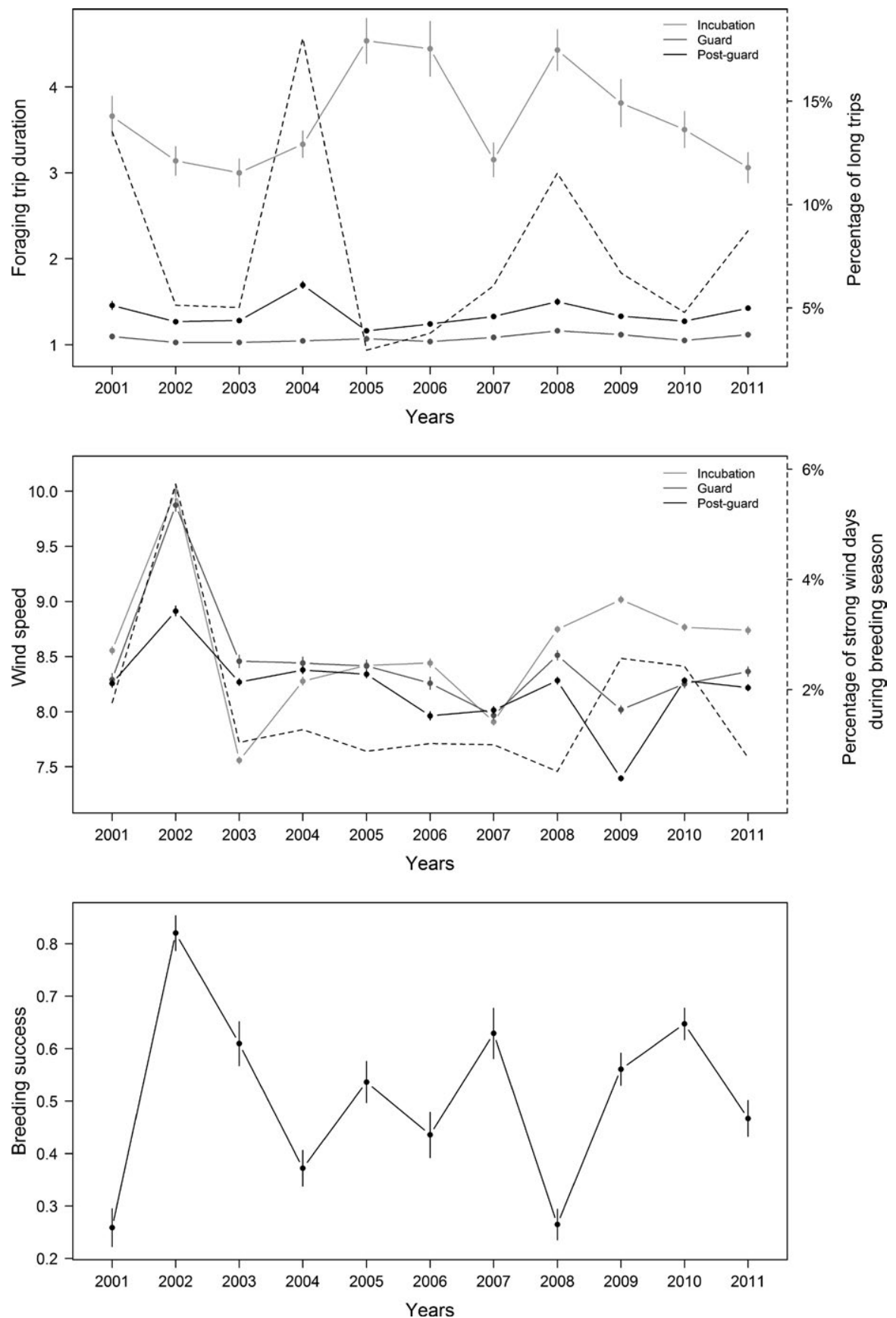

FIG. 1. Summary figure of the data presenting foraging trip duration (in days) and wind speed (in $\mathrm{m} / \mathrm{s}$ ) during each breeding stage, and breeding success of the colony from 2001 to 2011. Mean ( \pm SEM) per year. In the first two panels, the dotted lines represent percentages of long trips and strong wind days respectively, the $y$-axis being on the right. 
had different influences on the colony. For instance, wind conditions early or late in the season when only a few penguins are breeding may be less important than during the peak of the breeding season. Thus, we described wind annual statistics (Fig. 1 and Supplementary Materials, Fig. S1) by weighting daily wind variables by the number of breeding penguins passing over the APMS per day. In other words, a daily value of wind speed was repeated as many times as there were penguins crossing on this day, before averaging values over the breeding season. Similarly, wind statistics per breeding stage were estimated by weighting daily wind variables by the number of crossing penguins that were at this breeding stage on a given day.

\section{Statistics}

Statistics were done in R v.2.15.2 (R Development Core Team 2014). Data on foraging trips and mass parameters (body mass gain, meal size, and reserve changes) were longitudinal as individual penguins performed several trips in a breeding season and individual penguins were monitored over multiple breeding seasons. Data were modeled using a mixed model approach with individual and year as random effects to account for repeated measures. Generalized Lineal Mixed Models (GLMMs) were fitted with a Poisson distribution (log link function) for foraging trip duration, and Linear Mixed Models (LMMs) were used for all three mass parameters. The effect of wind speed on foraging and mass parameters was studied both continuously (wind speed in $\mathrm{m} / \mathrm{s}$ and wind speed ${ }^{2}$ to account for potential quadratic effects) and categorically (usual vs. strong wind conditions). For each model, the effects of wind speed, wind speed ${ }^{2}$ (or wind speed category), and their interactions with breeding stage and bird sex were considered. Akaike's Information Criterion (AIC) was used to compare candidate models fitted by Maximum Likelihood, and we retained models with the lowest AIC and highest AIC weight $\left(w_{i}\right.$, i.e. probability of being the best model among those presented) as best models. When competing models were close $(\triangle \mathrm{AIC}<2)$, we retained the most parsimonious model with the lowest number of explanatory variables (Burnham and Anderson 2002). Final models were fitted by Restricted Maximum Likelihood in order to get most accurate estimates. Whenever an interaction term was retained in the best model, separate models were run for each level of the considered factor (breeding stage, sex).

Data on pair breeding success were obtained over multiple breeding seasons, and a GLMM approach (binomial distribution, logit link function) was used, specifying both partner identities and year as random variables. Pair breeding success was analyzed by comparing the number of laid eggs that fledged to the number of laid eggs that failed. First, we analyzed the effects of wind speed on breeding success. Second, to help understand the mechanisms by which breeding success was impacted, we investigated the joint effects of foraging parameters (foraging-trip duration, body mass changes, reserve gain, and chick meal size) on breeding success. Foraging-trip duration was considered during incubation, guard, and post-guard. Body mass gain was considered during incubation only and separated in chick meal size and adult reserve changes for the guard and post-guard periods. Foraging and wind parameters were averaged by individual and breeding season, so that a single representation of these variables could be related to breeding success. This was preferred to retaining all records, which would artificially inflate degrees of freedom and increase the chance of Type II errors. All variables were standardized before inclusion in the GLMM so that model estimates were comparable. Variance inflation factors of independent variables were comprised between 1.03 and 1.18 (suggested cut-off about 10; Myers 1990), indicating no significant bias due to collinearity (Zuur et al. 2010). The effect of wind speed and percentage of strong wind days on the annual breeding success of the colony was investigated using Generalized Linear Models (GLMs) fitted with binomial distributions.

Variability in wind speed from the Cape Otway dataset from 1861 to 2013 was investigated using time series analysis. Data were analyzed using (1) monthly means and (2) annual quantiles to better represent the richness of the dataset and to remove effects of seasonality. First, whether wind speed was stationary over time was assessed using a linear model that provided an estimated slope of the regression with time. In time-series analyses, a stationary process is a stochastic process whose joint probability distribution does not vary through time. As a consequence, the mean of a stationary process does not change over time and does not follow any trend (Priestley 1981). Due to the autocorrelation and periodicity present in the time series of monthly wind speed, the significance of this estimate was assessed by comparing it to 10000 estimates obtained from 10000 simulated Fourier surrogates (datasets with the same statistical properties, in particular same autocorrelation and periodicity structures). Further, periodicity was investigated through spectral density analysis and the time series was later decomposed according to an addition of trend, seasonal, and random signals.

Model outputs are provided \pm standard errors (SE). The number of observations is given as $n$, while the number of individuals or pairs concerned is given as $N$. In the figures, for representation and clarity, the 17363 individual foraging trip data points were represented as averages $\pm \mathrm{SE}$ in $2 \mathrm{~m} / \mathrm{s}$ wind speed bins. However, analyses were run on the continuous (not categorically binned) data.

\section{RESUlts}

Description of variability in wind conditions, foraging trip durations, and breeding success

Over the 11 breeding seasons, we recorded substantial variation in foraging trip duration, daily wind speed, and breeding success (Fig. 1). Foraging trip dura- 
tion varied significantly among breeding seasons both during incubation (GLMM Poisson; $\triangle \mathrm{AIC}=-124$ with the null model) and post-guard (GLMM Poisson; $\triangle \mathrm{AIC}=-134$ with the null model), but not during chick guard (GLMM Poisson; $\triangle \mathrm{AIC}=13$ with the null model). The proportion of long trips ( $\geq 3 \mathrm{~d}$ ) that occurred during post-guard also varied substantially, ranging from $3 \%$ in 2005 to $18 \%$ in 2004 . Similarly, daily wind speed and the proportion of strong wind days varied significantly among breeding seasons (Fig. 1; LMM: $\triangle \mathrm{AIC}=-597$ with the null model for daily wind speed; LM: $P<0.001$ for proportion of strong wind days). On average, daily wind speed decreased within the breeding season. It was lower during post-guard $(8.17 \pm 0.01 \mathrm{~m} / \mathrm{s})$ than during guard $(8.41 \pm 0.01 \mathrm{~m} / \mathrm{s})$ and lower during guard than during incubation $(8.61 \pm 0.01 \mathrm{~m} / \mathrm{s} ; t$ tests; all $P<0.001)$. In all breeding seasons and regardless of breeding stage, winds blowing from the west or southwest were both dominant (in terms of number of events) and stronger than winds blowing from other directions (see Supplementary Materials, Fig. S2).

Breeding took place in the austral spring and summer, and mean laying date varied from September (2012) to November (2003). The breeding success of the colony varied substantially over the 11 breeding seasons, ranging from $34 \%$ in 2001 to $90 \%$ in 2002 (GLMM binomial: $\triangle \mathrm{AIC}=-79$ with the null model; Fig. 1$)$.

\section{Effects of wind speed on foraging trip duration}

The best and most parsimonious model explaining foraging trip duration contained sex, stage, speed category, and the interaction breeding stage $\times$ speed category (Table 1). Running separate models for each breeding stage showed that during incubation, sex, but not wind speed category, was an important factor explaining foraging trip duration. Indeed, males made shorter foraging trips than females $(3.18 \pm 0.01$ vs. $4.03 \pm 0.01 \mathrm{~d}$, Table 1). During guard, neither sex nor wind speed category appeared to explain foraging trip duration (Table 1; Fig. 2A, E). In contrast, wind speed category was an important factor explaining foraging trip duration during post-guard (Table 1). Foraging trips were $28 \%$ longer when they occurred in strong compared to usual wind conditions $(1.71 \pm 0.09 \mathrm{~d}$ vs. $1.34 \pm 0.01 \mathrm{~d}$; Table 1, Fig. 2E). This resulted in the higher occurrence of long foraging trips ( $\geq 3 \mathrm{~d}$ ) when winds were strong, i.e. $17.5 \%$ vs. $7.4 \%$ in usual wind conditions.

\section{Effects of wind speed on adult body mass gain, chick meal size, and adult body reserves}

For all mass parameters (adult body mass gain, chick meal size, and reserve changes), the best model included the interaction between breeding stage and a wind parameter (either wind speed and its quadratic form wind speed $^{2}$ or wind speed category) as important explanatory variables (Tables $2-4$ ). Thus, we ran sepa- rate models for each breeding stage. In all three stages, adult body mass gain was higher in males than females $(227 \pm 4$ g vs. $213 \pm 4$ g, $273 \pm 3$ g vs. $257 \pm 3$ g, $275 \pm 2$ g vs. $250 \pm 2 \mathrm{~g}$, in incubation, guard, and post-guard respectively; Table 2). Adult body mass gain during a foraging trip decreased with increasing wind speed both during incubation (LMM; linear slope $=-3.07 \pm 1.22$; Table 2) and guard (quadratic effect: $-0.20 \pm 0.05$; Table 2), but not during post-guard (see Fig. 2B). Chick meal size decreased with increasing squared wind speed during guard (LMM; quadratic effect: $-0.22 \pm 0.05$; Table 3) and was lower when winds were strong during post-guard, i.e. $241 \pm 10 \mathrm{~g}$ vs. $263 \pm 1 \mathrm{~g}$ for usual wind conditions; Table 3, Fig. 2C, G). In addition, during post-guard, chick meal size was smaller when provided by females $(249 \pm 2 \mathrm{~g}$ vs. $276 \pm 2 \mathrm{~g}$ for males $)$. Finally, reserve changes were not influenced by sex or wind speed during guard (Table 4, Fig. 2D, H), but wind speed appeared to be an important factor affecting reserve changes during post-guard. Indeed, both the linear $(-10.51 \pm 3.33)$ and quadratic $\left(0.62 \pm 0.18\right.$ wind $\left.^{2}\right)$ terms of wind speed were retained in the best model (Table 4). Reserve changes first decreased and subsequently increased with increasing wind speed (Fig. 2D).

\section{Effects of wind and foraging parameters on breeding success}

Pair breeding success decreased with increasing average daily wind speed over the breeding season (GLMM with a binomial distribution; slope $=-0.31 \pm 0.12$; $\left.n=726, N=173 / 170 ; w_{i}=0.91\right)$. However, the average daily wind speed over the season did not explain the average breeding success of the colony (GLM: $P>0.30$, $n=11)$.

The best and most parsimonious model explaining variation in pair breeding success retained foraging trip duration during post-guard, and chick meal size during guard and post-guard (GLMM with a binomial distribution; $w i=0.10, n=276, N=94 / 81$ ). Foraging trip duration during post-guard had a negative effect on pair breeding success, with longer trips causing lower breeding success (slope $=-0.41 \pm 0.18$; see Fig. 3 ). Chick meal size had a positive effect on pair breeding success both during guard $(0.32 \pm 0.12)$ and post-guard $(0.47 \pm 0.14)$, with larger meals resulting in a higher probability of fledging the chicks (Fig. 3). In contrast, incubation variables (foraging trip duration and body mass gain) and reserve changes during chick rearing did not appear to importantly affect pair breeding success (Fig. 3).

\section{Wind patterns from 1861-2013}

Monthly wind speed measured at the Cape Otway wind station decreased significantly over a period of $153 \mathrm{yr}$ (by $0.06 \mathrm{~m} / \mathrm{s}$ per $100 \mathrm{yr}$; LM; $a=-5.710^{-4}$, $P=0.03$ from comparisons with 10000 estimates on simulated time series of similar properties). The 


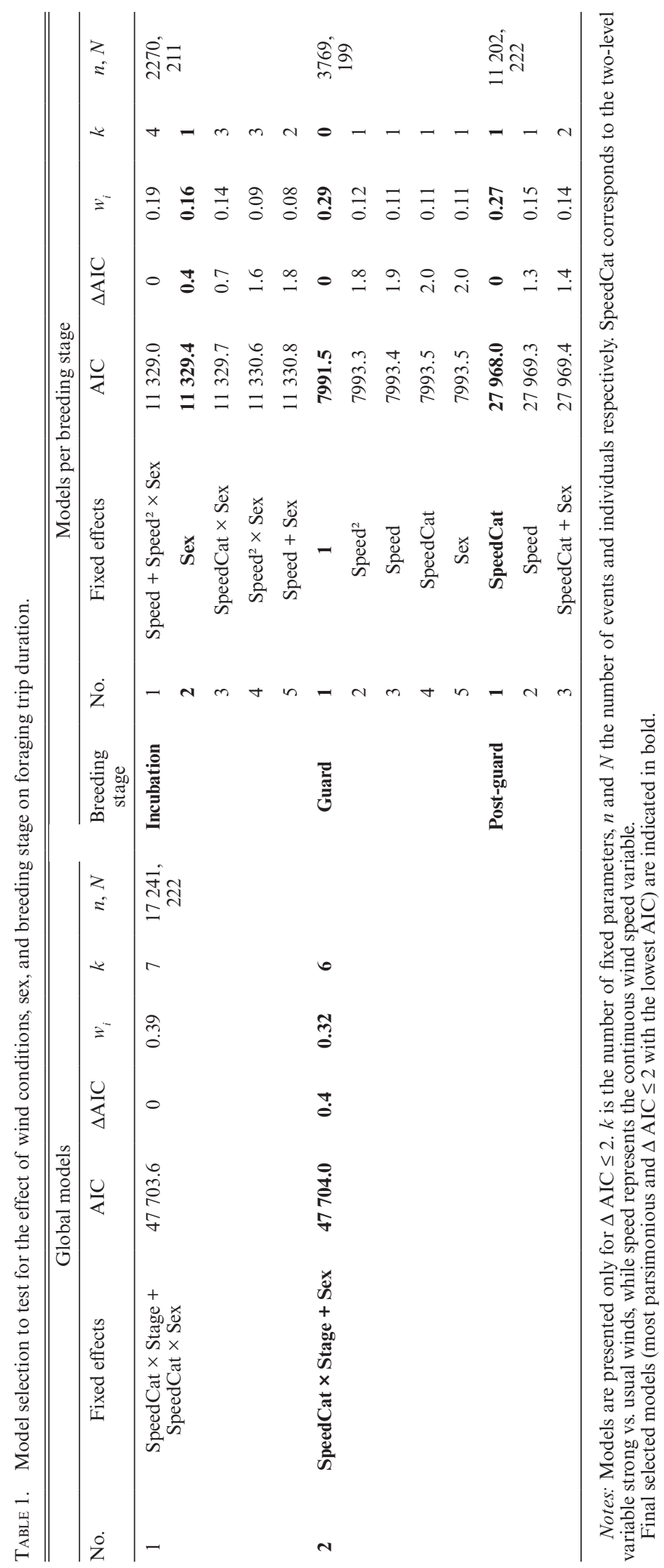




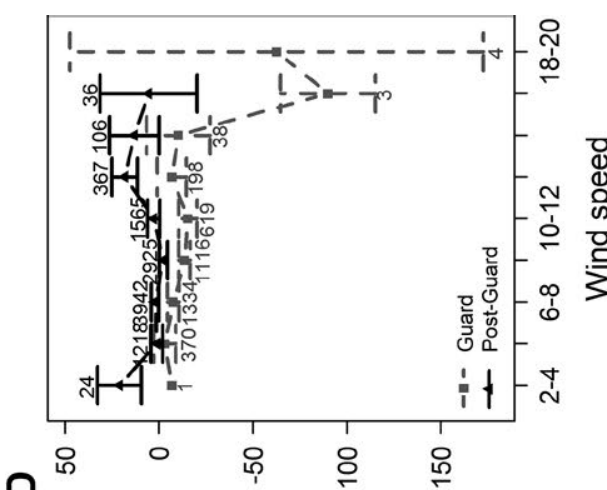

(b) sәбиечว әмјәsәу

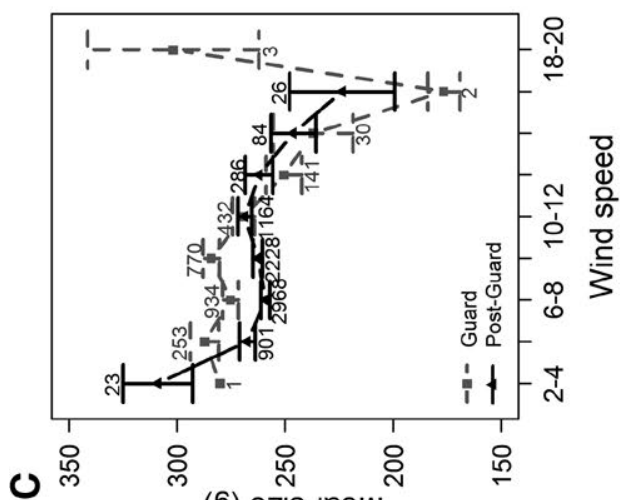

(6) əZ!̣ | $\mid e ә W$

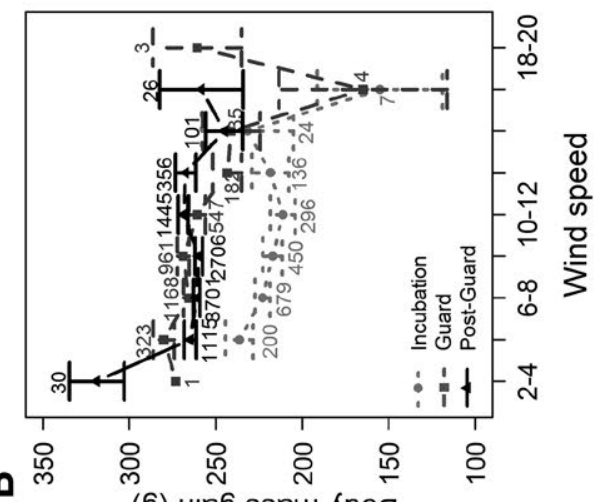

(6) u!̣e6 ssem кpog

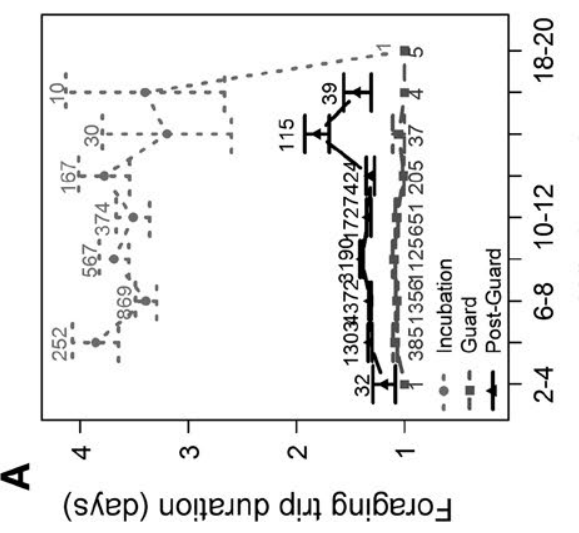

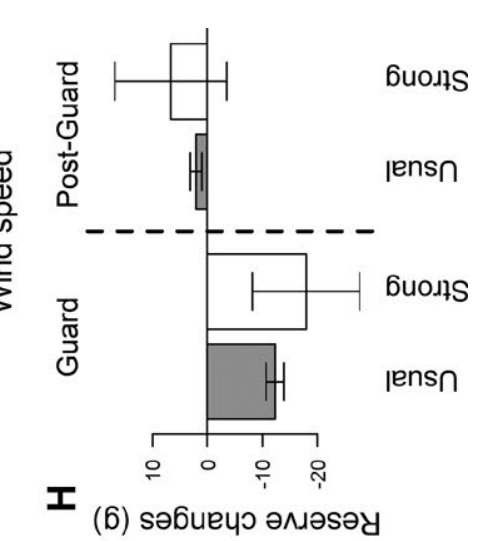

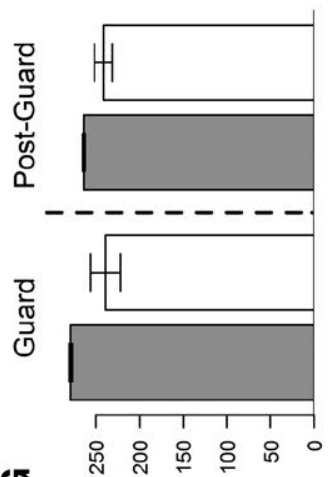

buods

jens?

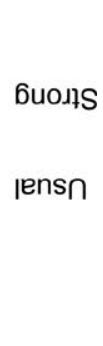

(6) əz!̣ ןeəW

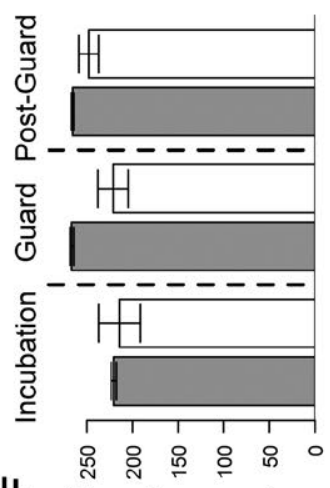

buods

ןens?

buods

jens?

6uods

jens?

(6) u!̣e6 ssem Kpog

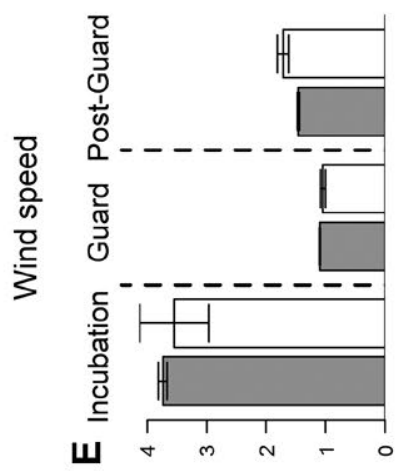

buods

jens?

(a)

buods

jens?

jens ก

buods

菏.

jens?

吾

凷的吾

$i++\infty$

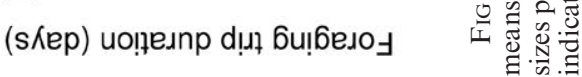




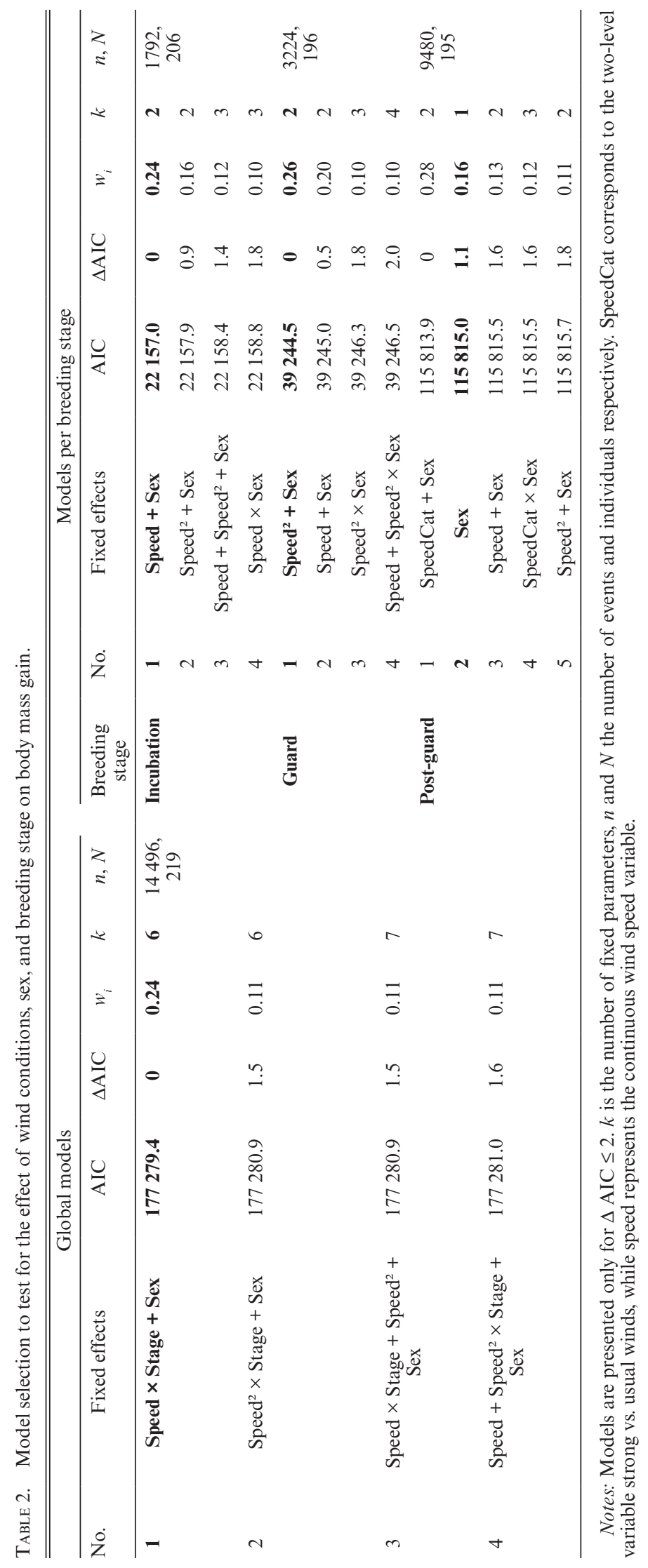




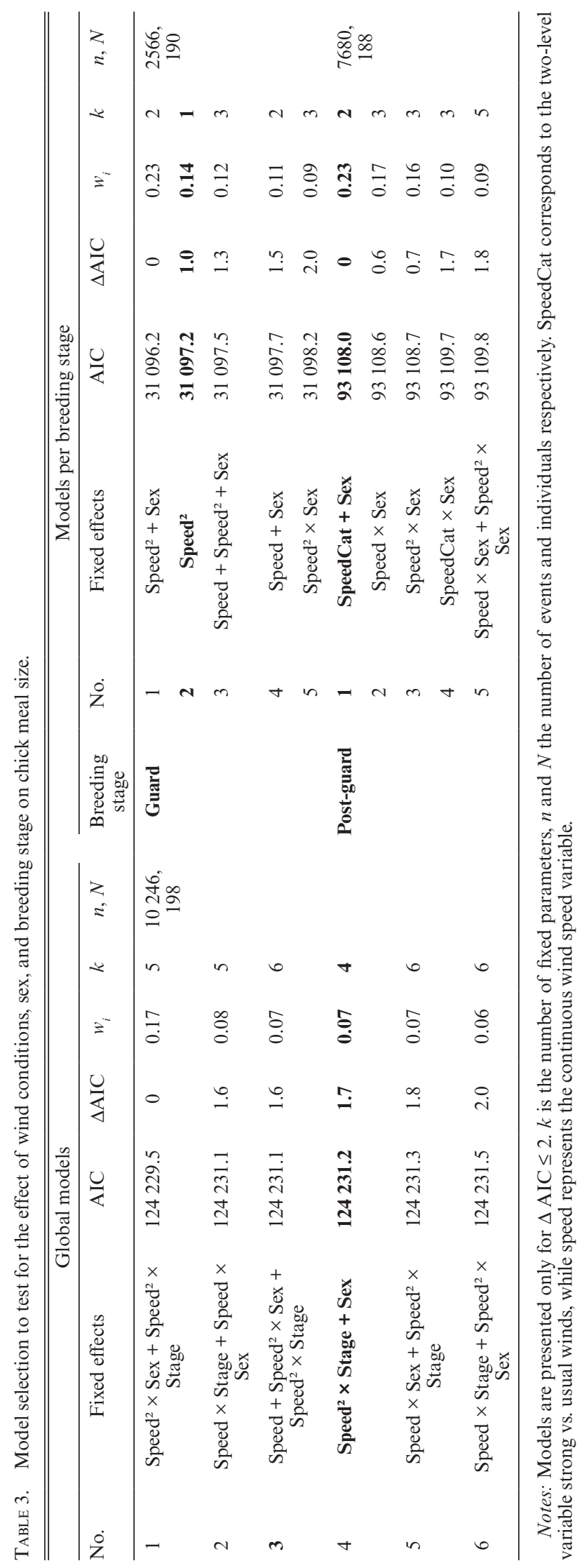




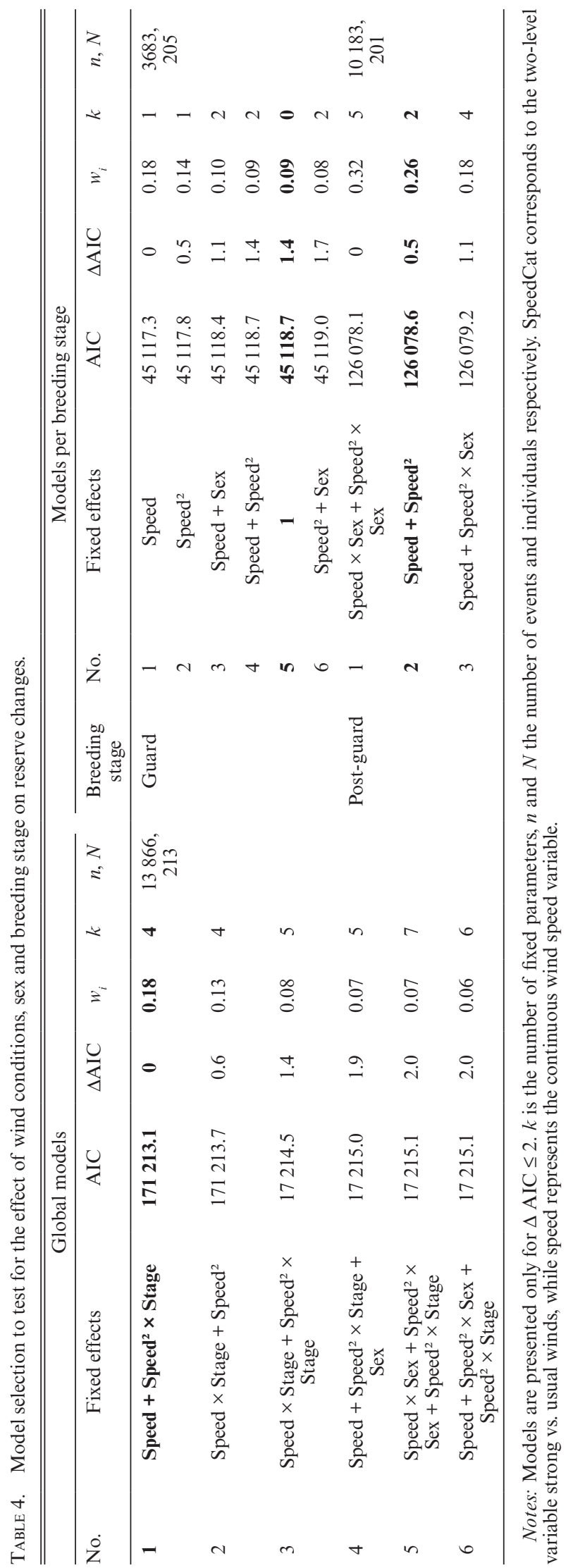



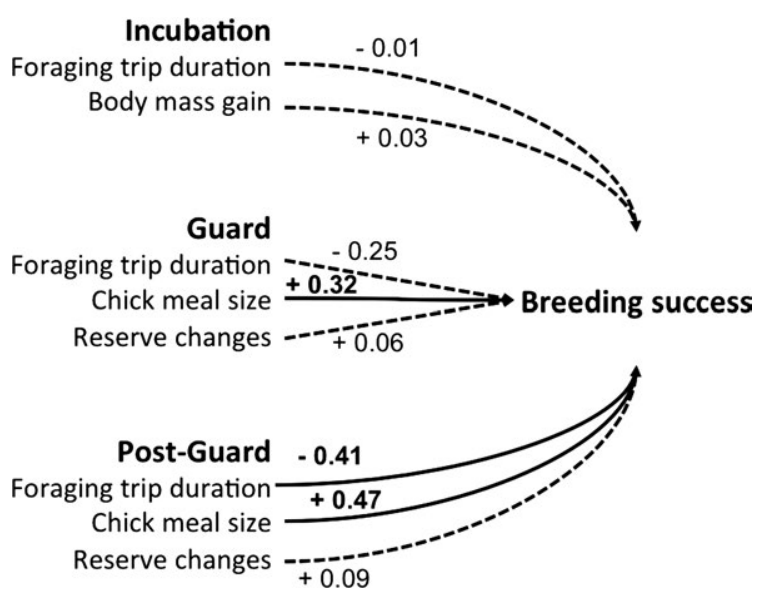

FIG. 3. Joint effects of foraging trip parameters on breeding success in little penguins. All variables were standardized and analyzed in the same model, so that path coefficients are directly comparable. Variance inflation factors ranged from 1.03 to 1.18 , indicating no significant bias due to collinearity. Factors retained in the best model are indicated by bold lines, and non-retained paths by dashed-lines.

estimate being very low, we still considered nonstationarity to be small and estimated its spectral density. We found an important peak at a period of 12 months, suggesting a 12-month periodicity in wind data as expected in temperate latitudes (Jakob 2010, L. Chambers, personal communication). As seasonal fluctuations were fairly constant over time, this seasonality was described using an additive model. The original time series as well as the additive decomposition of the signal in trend, seasonal, and random signals are presented in Supplementary Materials,
Fig. S2. Trend variability as well as random noise amplitude showed a clear decrease with time. Similarly, analyses of annual quantiles revealed a decrease in wind speed variability with time, the inter-annual variability being much smaller since the 60 s for the mean trend and each investigated quantile (Fig. 4A). The amplitude of the $95 \%$ data interval (difference between the 2.5 and $97.5 \%$ quantiles) also decreased through time (LM: slope $=-0.06 \pm 0.00, P<0.001$; Fig. 4B). Finally, the percentage of strong wind days per year measured at the Cape Otway wind station decreased significantly over a period of $153 \mathrm{yr}$ (by 7 percentage points per $100 \mathrm{yr}$; LM; $a=-0.074$, $P<0.001)$.

\section{Discussion}

Over 11 breeding seasons, involving more than 17000 foraging trips from 222 breeding birds, we showed that wind speed substantially affected the behavior, body condition, and breeding success of little penguins. Strong winds had varying consequences on the length of adult foraging trips, body mass gain, chick meal size, and adult reserve gain, depending on the breeding stage.

During incubation and chick-guard (but not postguard) adult body mass gain from foraging trips decreased with increasing wind speed. Similar effects of wind speed on foraging success were recently reported for rockhopper penguins Eudyptes chrysocome (Dehnhard et al. 2013), suggesting that strong wind conditions associated with punctual storms may render foraging generally more challenging for penguins (as described later in the Discussion). These negative effects could be driven by several non-mutually
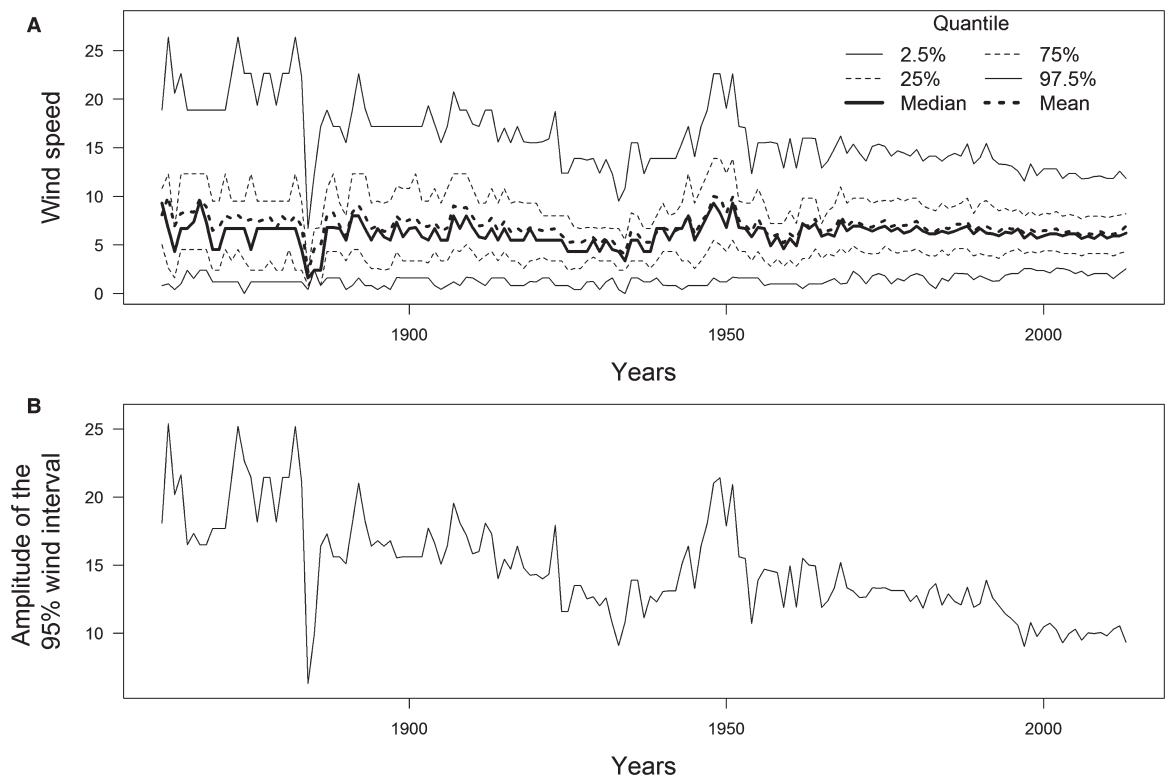

FIG. 4. Annual wind speed time series (1861-2013). Panel (A) shows the different quantiles $(2.5 \%, 25 \%, 50 \%, 75 \%$, and $97.5 \%)$ as well as the mean time series. Panel (B) shows the decrease in amplitude of the $95 \%$ interval. 
exclusive mechanisms. Strong winds could affect birds' foraging effort (Lewis et al. 2015) and success by physically mixing water masses, or disrupting thermal stratification and rendering the prey harder to locate. For instance, ectothermic fish are known to concentrate around thermoclines (Sogard and Olla 1993, Hansen et al. 2001), suggesting that diving seabirds may be able to target and take advantage of areas of high prey-encounter rates (Charrassin and Bost 2001). There is some evidence that thermoclines are indeed important cues for prey location in seabirds (Kokubun et al. 2010), including little penguins (Ropert-Coudert et al. 2009, Pelletier et al. 2012). However, oceanographic mixing models have shown that thermoclines are relatively resilient to the action of wind in the Bass Strait (Jones 1980, Fandry 1982), and whether wind may actually affect thermoclines in the nutrient rich, shallower $(20-30 \mathrm{~m})$ regions of the strait (Gibbs 1992) that may be used by little penguins during foraging bouts (Hoskins et al. 2008, Preston et al. 2010, Kowalczyk et al. 2015) remains to be thoroughly investigated. In addition, changes in oceanographic conditions related to wind could also have affected the flow and concentration of nutrients in the Bass Strait (Gibbs 1992), resulting in changes of prey distribution. Locating prey may thus have been harder when winds were strong, affecting the diving energetics of parents (e.g. as in the common eider, Somateria mollissima sedentaria; Heath and Gilchrist 2010). Alternately, wind may substantially impact the energy cost of travelling between nesting and foraging areas (Weimerskirch et al. 2000, Amélineau et al. 2014, Elliott et al. 2014). This may seem obvious for flying seabirds, where the energy cost of flying is expected to increase when birds are moving against the wind. For instance, thick-billed murres (Uria lomvia) and blacklegged kittiwakes (Rissa tridactyla) are known to substantially increase wing beat frequency and instantaneous energy costs when facing high headwinds (Elliott et al. 2014). For flightless penguins, on top of a potential direct effect of wind speed on locomotion during surface travels, a consequent indirect effect may be mediated by the formation of large surface waves (Bailey et al. 1991, Young et al. 2011), which could hinder efficient navigation. For instance, Berlincourt and Arnould (2015) have recently shown that little penguins in Southeast Australia spend less time diving and foraging in rough seas (high waves and strong wind conditions), a result similar to what was found in European shags (Lewis et al. 2015). On Phillip Island, breeding penguins have been reported to forage west (Weavers 1992, Collins et al. 1999) and east (Pelletier et al. 2014) of the colony. Given that winds, particularly when strong, blew predominantly from the west in our study, penguins could well be working harder on their outbound (penguins foraging west of Phillip Island) or inbound (penguins foraging east of Phillip Island) journey. It would be particu- larly insightful to combine measures of energy costs of foraging with predictive modeling of wave formation and propagation in varying wind regimes to better understand the extent to which the interaction between wind and swell formation may affect penguin energetics. Strong winds are also likely to augment the thermoregulatory costs of endothermy (Stahel et al. 1986), affecting body mass loss. Our current capacity to estimate the energy costs of travelling in windy conditions using accelerometers (e.g. Elliott et al. 2014) or doubly-labeled water, coupled with the increasing predictive accuracy of wave modeling (Cavaleri et al. 2007), certainly opens exciting perspectives for future research on determining the role of wind on seabird behavior and energetics.

Importantly, changes in foraging efficiency under strong wind conditions should result in a modulation of the energy available to allocate to maintenance vs. reproduction (van Noordwijk and de Jong 1986). As individual maintenance is a trait contributing more strongly than reproduction to individual fitness in long-lived species (Stearns 1992), little penguins should prioritize individual maintenance over reproduction when they can no longer cope with energy limitations due to the environment. As such, a decrease of available energy should translate into a change of strategy for adults, especially during energetically demanding chick rearing (Gales and Green 1990). Foraging-trip duration was not affected by wind conditions during chick-guard in our study. This could be explained by the fact that during this period, penguins are constrained by having to relieve their fasting partner at the nest and are perhaps less flexible in their foraging strategies than during post-guard (Chiaradia and Nisbet 2006). The decrease in body mass gain with increasing wind speed during this period resulted in a decrease of chick meal size (especially when winds were stronger, i.e. quadratic effect), but did not affect adult body reserves. During post-guard, the chick is left unattended, and the amount of time spent foraging may increase (Saraux et al. 2011a). The energy costs of raising chicks are especially exacerbated during late chick rearing (e.g. the field metabolic rate of adult little penguins increases by $100.8 \%$ between early- and late-chick rearing; Gales and Green 1990). At this critical stage, adults increased foraging trip duration and the proportion of long-trips under extreme wind conditions. Whereas body mass gain remained stable with increasing winds over this period, chick meal size also decreased under extreme wind conditions, and adult reserve change followed a U-shaped quadratic relationship with increasing wind speed (decreasing at first before increasing again for extreme wind speeds). In little penguins, long trips are undertaken to restore adult body condition, whereas short trips are used to provision the chicks (Saraux et al. 2011a). Taken together, those results are consistent with life-history predictions (Stearns 1992, Roff 
2002) and suggest that when long-lived little penguins are no longer able to cope with extreme environmental constraints, parents may change allocation strategies, shifting environmental costs towards their offspring and safeguarding their own body reserves. Finally, although males and females differed in a number of parameters (e.g. males gained more body mass during a foraging trip than females in all breeding stages), no important sex $\times$ wind interaction was found in our models, suggesting that both sexes coped similarly with adverse wind conditions.

Earlier studies hypothesized a connection between wind patterns and breeding success in little penguins (Mickelson et al. 1991), and recent findings have revealed negative effects of easterly summer winds on little penguin adult survival, the other main component of individual fitness (Ganendran et al. 2011). In our study, the effect of wind speed on individual breeding success seems to be mediated through limitations of foraging performance during late chick rearing. Breeding success was little affected by foraging trip duration and changes in adult body mass during incubation, but was strongly affected by trip duration and chick meal size during chick rearing (less so during guard than post-guard). Thus, by altering chick meal size and extending foraging trip duration during chick rearing, strong winds had a net negative effect on breeding success.

Despite the wind effect at an individual level, the average wind speed during a breeding season was not related to variability in annual breeding success of the overall colony. We cannot exclude the possibility that this apparent conflict might be the result of low sample size and statistical power $(n=11$ breeding seasons, i.e. 11 data points). However, it might also be associated with other interesting phenomena. First, we saw that wind effects were immediate and occurred mostly during the critical chick-rearing phase, so averages at the entire breeding season scale might smooth and overlook any meaningful biological pattern. Also, several demographic processes can lead to various responses at the individual and population levels (e.g. density dependence; Reed et al. 2012). A previous study demonstrated the consistency of individual foraging performance in little penguins (Saraux et al. 2011b), so that looking at the role of individual quality to modulate susceptibility to environmental conditions should help in understanding the differences between individual and population responses.

Given the above, the question is then whether little penguins are at risk facing climate change and predicted increases in extreme weather events (Solomon et al. 2007). An increase in wind speed in the southern hemisphere was linked to increased fitness in the wandering albatross Diomedea exulans (Weimerskirch et al. 2012), suggesting very different effects on soaring vs. diving seabirds (as in our study). In Australia, the $150 \mathrm{yr}$ of wind data collected in the breeding area of our penguin population revealed that wind speed and variability in speed have been decreasing over time. Although part of this change in variability might be due to the change from manual to automatic weather stations, the scenario under which little penguin breeding success could be impacted by a strengthening of wind in the future (Cai et al. 2005) seems unlikely. Rather, our data suggests that these inshore breeders are more sensitive to punctual strong wind events for which they are able to compensate via plasticity in foraging strategies (Saraux et al. 2011a), at least up to a point. A better understanding of the limits in behavioral plasticity is required to enable improved predictions on future climate impacts in little penguins.

\section{ACKNOWLEDGMENTS}

We are grateful for all honors students who assisted on the data collection over the 11-yr period: S. Robinson-Laverick, T. Daniel, P. Fallow, P. Wasiak, R. Long, H. Davies, D. Steirt, M. Simpson and D. Hedger. Land-based wind data originated from Bureau of Meteorology, Australia. The Australian Antarctic Division kindly provided the automated penguin monitoring system (APMS). We are indebted to K. Kerry, K. Newbery, K. Maizels and all engineers involved in the research and development of the APMS. We thank the continuous support of the Phillip Island Nature Parks and Monash University. This project was supported by several grants from Penguin Foundation, Australian Academy of Science, and AAT Kings. V. A. Viblanc was the recipient of an AXA Research Fund post-doctoral fellowship. The project was approved by the Phillip Island Nature Parks Animal Experimentation Ethics Committee and carried a research permit issued by the Department of Environment, Land, Water and Planning of Victoria, Australia.

C. Saraux, A. Chiaradia, M. Salton, P. Dann, and V. A. Viblanc designed the study; A. Chiaradia, M. Salton, and C. Saraux collected the data; M. Salton pre-analyzed the data; C. Saraux, A. Chiaradia, and V. A. Viblanc analyzed the data; V. A. Viblanc and C. Saraux wrote the manuscript. All authors commented on the manuscript.

\section{Literature Cited}

Afán, I., A. Chiaradia, G. Forero Manuela, P. Dann, and F. Ramírez. 2015. A novel spatio-temporal scale based on ocean currents unravel environmental drivers of reproductive timing in a marine predator. Proceedings of the Royal Society of London B: Biological Sciences 282:20150721.

Amélineau, F., C. Péron, A. Lescroël, M. Autthier, P. Provost, and D. Grémillet. 2014. Windscape and tortuosity shape the flight costs of northern gannets. Journal of Experimental Biology 217:876-885.

Arnould, J. P. Y., P. Dann, and J. M. Cullen. 2004. Determining the sex of little penguins (Eudyptula minor) in northern Bass Strait using morphometric measurements. Emu 104:261-265.

Bailey, R. J., I. Jones, and Y. Toba. 1991. The steepness and shape of wind waves. Journal of the Oceanographical Society of Japan 47:249-264.

Bale, J. S., et al. 2002. Herbivory in global climate change research: direct effects of rising temperature on insect herbivores. Global Change Biology 8:1-16.

Berlincourt, M., and J. P. Y. Arnould. 2015. Influence of environmental conditions on foraging behavior and its consequences 
on reproductive performance in little penguins. Marine Biology 162:1485-1501.

Bize, P., A. Stocker, S. Jenni-Eiermann, J. Gasparini, and A. Roulin. 2010. Sudden weather deterioration but not brood size affects baseline corticosterone levels in nestling Alpine swifts. Hormones and Behavior 58:591-598.

Boyd, I. L., and A. W. A. Murray. 2001. Monitoring a marine ecosystem using responses of upper trophic level predators. Journal of Animal Ecology 70:747-760.

Burnham, K. P., \& Anderson, D. R. 2002. Model selection and multimodel inference: a practical information-theoretic approach. Springer Science \& Business Media.

Cai, W., G. Shi, T. Cowan, D. Bi, and J. Ribbe. 2005. The response of the southern annular mode, the east Australian current, and the southern mid-latitude ocean circulation to global warming. Geophysical Research Letters 32:L23706.

Cannell, B. L., and J. M. Cullen. 1998. The foraging behaviour of little penguins Eudyptula minor at different light levels. Ibis 140:467-471.

Carol, E. A., and B. C. Congdon. 2007. Day-to-day variation in sea-surface temperature reduces sooty tern Sterna fuscata foraging success on the Great Barrier Reef, Australia. Marine Ecology Progress Series 331:255-266.

Cavaleri, L., et al. 2007. Wave modelling - the state of the art. Progress in Oceanography 75:603-674.

Charrassin, J. B., \& Bost, C. A. 2001. Utilisation of the oceanic habitat by king penguins over the annual cycle. Marine Ecology Progress Series, 221:285-298.

Chiaradia, J. B., and K. R. Kerry. 1999. Daily nest attendance and breeding performance in the Little Penguins Eudyptula minor at Phillip Island, Australia. Marine Ornithology 27:13-20.

Chiaradia, A., \& Bost, C. A. 2001. Utilisation of the oceanic habitat by king penguins over the annual cycle. Marine Ecology Progress Series, 221:285-298.

Chiaradia, A., and I. C. T. Nisbet. 2006. Plasticity in parental provisioning and chick growth in Little Penguins Eudyptula minor in years of high and low breeding success. Ardea 94:257-270.

Chiaradia, A., J. McBride, T. Murray, and P. Dann. 2007. Effect of fog on the arrival time in little penguins Eudyptula minor: a clue for visual orientation? Journal of Ornithology 148:229-233.

Chiaradia, A., M. G. Forero, K. A. Hobson, and J. M. Cullen. 2010. Changes in diet and trophic position of a top predator ten years after a mass mortality of a key prey. ICES Journal of Marine Science 67:1710-1720.

Collins, M., J. M. Cullen, and P. Dann. 1999. Seasonal and annual foraging movements of Little Penguins from Phillip Island, Victoria. Wildlife Research 26:705-721.

Daniel, T. A., A. Chiaradia, M. Logan, G. Quinn, and R. Reina. 2007. Synchronized group association in little penguins, Eudyptula minor. Animal Behaviour 74:1241-1248.

Dann, P., J. M. Cullen, R. Thoday, and R. Jessop. 1992. Movements and patterns of mortality at sea of little penguins Eudyptula minor from Phillip Island, Victoria. Emu 91:278286.

Dehnhard, N., K. Ludynia, M. Poisbleau, L. Demongin, and P. Quillfeldt. 2013. Good days, bad days: wind as a driver of foraging success in a flightless seabird, the southern rockhopper penguin. PLoS One 8:e79487.

Elliott, K. H., et al. 2014. Windscapes shape seabird instantaneous energy costs but adult behavior buffers impact on offspring. Movement Ecology 2:17.

Fairbanks, B., and F. S. Dobson. 2007. Mechanisms of the group-size effect on vigilance in Columbian ground squirrels: dilution versus detection. Animal Behaviour 73:115-123.

Fandry, C. B. 1982. A numerical model of the wind-driven transient motion in Bass Strait. Journal of Geophysical Research $87: 499-517$
Forcada, J., P. N. Trathan, and E. J. Murphy. 2005. The effects of global climate variability in pup production of Antarctic fur seals. Ecology 86:2408-2417.

Forchhammer, M. C., E. Post, and N. C. Stenseth. 1998. Breeding phenology and climate. Nature 391:29-30.

Gales, R., and B. Green. 1990. The annual energetics cycle of little penguins (Eudyptula minor). Ecology 71:2297-2312.

Ganendran, L. B., L. A. Sidhu, E. A. Catchpole, P. Dann, and L. E. Chambers. 2011. The effect of directional wind components on survival of Little Penguins Eudyptula minor. ANZIAM 52:1012-1030.

Garrison, T. S. 2011. Essentials of Oceanography, 6th edition. Brooks Cole Publishers, Pacific Grove, California, USA.

Gibbs, C. F. 1992. Oceanography of Bass Strait: implications for the food supply of little penguins Eudyptula minor. Emu 91:395-401.

Gjerdrum, C., A. M. J. Vallée, C. C. St. Clair, D. F. Bertram, J. L. Ryder, and G. S. Blackburn. 2003. Tufted puffin reproduction reveals ocean climate variability. Proceedings of the National Academy of Sciences of the United States of America 100:9377-9382.

Hansen, J. E., P. Martos, and A. Madirolas. 2001. Relationship between spatial distribution of the Patagonian stock of Argentine anchovy, Engraulis anchoita, and sea temperatures during late spring to early summer. Fish Oceanography 10:193-206.

Heath, J. P., and H. G. Gilchrist. 2010. When foraging becomes unprofitable: energetics of diving in tidal currents by common eiders wintering in the Arctic. Marine Ecology Progress Series 403:279-290

Hoskins, A. J., P. Dann, Y. Ropert-Coudert, A. Kato, A. Chiaradia, D. P. Costa, and J. P. Y. Arnould. 2008. Foraging behaviour and habitat selection of the little penguin Eudyptula minor during early chick rearing in Bass Strait, Australia. Marine Ecology Progress Series 366:293-303.

Hughes, L. 2000. Biological consequences of global warming: is the signal already apparent? Trends in Ecology \& Evolution 15:56-61.

Jakob, D. 2010. Challenges in developing a high-quality surface wind-speed data-set for Australia. Australian Meteorological and Oceanographic Journal 60:227-236.

Jones, I. 1980. Tidal and wind-driven currents in Bass Strait. Australian Journal of Marine and Freshwater Research 31:109-117.

Kappelle, M., M. M. I. Van Vuuren, and P. Baas. 1999. Effects of climate change on biodiversity: a review and identification of key research issues. Biodiversity and Conservation 8:1383-1397.

Kerry, K., J. Clarke, and G. Else. 1993. The use of an automated weighing and recording system for the study of the biology of Adélie penguins Pygoscelis adeliae. Pages 62-75 in Proceedings of National Institute of Polar Research Symposium on Polar Biology, Tokyo, Japan.

Kitaysky, A. S., and E. G. Golubova. 2000. Climate change causes contrasting trends in reproductive performance of planktivorous and piscivorous alcids. Journal of Animal Ecology 69:248-262.

Klein, P., and B. Coste. 1984. Effects of wind-stress variability on nutrient transport into the mixed layer. Deep Sea Research Part A 31:21-37.

Klomp, N. L., and R. D. Wooller. 1991. Patterns of arrival and departure by breeding little penguins at Penguin Island, Western Australia. Emu 91:32-35.

Kokubun, N., A. Takahashi, M. Ito, K. Matsumoto, A. S. Kitaysky, and Y. Watanuki. 2010. Annual variation in the foraging behavior of thick-billed murres in relation to upperocean thermal structure around St. Georges Island, Bering Sea. Aquatic Biology 8:289-298. 
Kowalczyk, N., R. Reina, T. J. Preston, and A. Chiaradia 2015. Environmental variability drives shifts in the foraging behaviour and reproductive success of an inshore seabird. Oecologia 178:967-979.

Lane, J. E., L. E. B. Kruuk, A. Charmantier, J. O. Murie, and F. S. Dobson. 2012. Delayed phenology and reduced fitness associated with climate change in a wild hibernator. Nature 489:554-557.

Le Bœuf, B. J., and D. E. Crocker. 2005. Ocean climate and seal condition. BMC Biology 3:9.

Lewis, S., R. A. Phillips, S. J. Burthe, S. Wanless, and F. Daunt. 2015. Contrasting responses of male and female foraging effort to year-round wind conditions. Journal of Animal Ecology 84:1490-1496. doi:10.1111/1365-2656.12419.

McCutcheon, C., P. Dann, M. Salton, L. Renwick, A. J. Hoskins, A. M. Gormley, and J. P. Y. Arnould. 2011. The foraging range of little penguins (Eudyptula minor) during winter. Emu 111:321-329.

Mickelson, M. J., P. Dann, and J. M. Cullen. 1991. Sea temperature in Bass Strait and breeding success of the little penguin Eudyptula minor at Phillip Island, South-Eastern Australia. Emu 91:355-368.

Myers, R. H. 1990. Classical and modern regression with applications. Second edition. PWS-Kent, Boston, Massachusetts, USA.

van Noordwijk, A. J., and G. de Jong. 1986. Acquisition and allocation of resources: their influence on variation in life history tactics. American Naturalist 128:137-142.

Palumbi, S. R., D. J. Barshis, N. Traylor-Knowles, and R. A. Bay. 2014. Mechanisms of reef coral resistance to future climate change. Science 344:895-898.

Parmesan, C. 2006. Ecological and evolutionary response to recent climate change. Annual Review of Ecology, Evolution, and Systematics 37:637-669.

Parmesan, C., T. L. Root, and M. R. Willig. 2000. Impacts of extreme weather and climate on terrestrial biota. Bulletin of the American Meteorological Society 81:443-450.

Pelletier, L., A. Kato, A. Chiaradia, and Y. Ropert-Coudert. 2012. Can thermoclines be a cue to prey distribution for marine top predators? A case study with little penguins. PLoS One 7:e31768

Pelletier, L., A. Chiaradia, A. Kato, and Y. Ropert-Coudert. 2014. Fine-scale spatial age segregation in the limited foraging area of an inshore seabird species, the little penguin. Oecologia 176:399-408.

Plard, F., J.-M. Gaillard, T. Coulson, A. J. M. Hewison, D. Delorme, C. Warnant, and C. Bonenfant. 2014. Mismatch between birth date and vegetation phenology slows the demography of roe deer. PLoS Biology 12:e1001828.

Preston, T. J., A. Chiaradia, S. A. Caarels, and R. D. Reina. 2010. Fine scale biologging of an inshore marine animal. Journal of Experimental Marine Biology and Ecology 390:196-202.

Priestley, M. B. 1981. Spectral analysis and time series. Academic Press, Waltham, Massachusetts, USA.

R Development Core Team. 2014. R: a language and environment for statistical computing. R Foundation for Statistical Computing, Vienna, Austria. http://www.R-project.org

Ramírez, F., M. G. Forero, K. A. Hobson, and A. Chiaradia. 2015. Older female little penguins Eudyptula minor adjust nutrient allocations to both eggs. Journal of Experimental Marine Biology and Ecology 468:91-96.

Reed, T. E., D. E. Schindler, and R. S. Waples. 2011. Integrating effects of phenotypic plasticity and evolution on population persistence in a changing climate. Conservation Biology 25:56-63.

Reed, T. E., S. Jenouvrier, and M. E. Visser. 2012. Phenological mismatch strongly affects individual fitness but not population demography in a woodland passerine. Journal of Animal Ecology 82:131-144
Robinson, S., A. Chiaradia, and M. A. Hindell. 2005. The effect of body condition on the timing and success of breeding in little penguins Eudyptula minor. Ibis 147:483-489.

Roff, D. A. 2002. Life history evolution. Sinauer Associates, Sunderland, Massachusetts, USA

Ropert-Coudert, Y., A. Kato, R. P. Wilson, and B. Cannell. 2006. Foraging strategies and prey encounter rate of free ranging little penguins. Marine Biology 149:139-148.

Ropert-Coudert, Y., A. Kato, and A. Chiaradia. 2009. Impact of small-scale environmental perturbations on local marine food resources: a case study of a predator, the little penguin. Proceedings of the Royal Society of London B: Biological Sciences 276:4105-4109.

Saraux, C., S. M. Robinson-Laverick, Y. Le Maho, Y. Ropert-Coudert, and A. Chiaradia. 2011a. Plasticity in foraging strategies of inshore birds: how little penguins maintain body reserves while feeding offspring. Ecology 92:1909-1916.

Saraux, C., A. Chiaradia, Y. Le Maho, and Y. Ropert-Coudert. 2011 b. Everybody needs somebody: unequal parental effort in little penguins. Behavioral Ecology 22:837-845.

Sogard, S. M., and B. L. Olla. 1993. Effects of light, thermoclines and predator presence on vertical distribution and behavioral interactions of juvenile walleye pollock, Theragra chalcogramma Pallas. Journal of Experimental Marine Biology and Ecology 167:179-195.

Solomon, S., D. Qin, M. Manning, Z. Chen, M. Marquis, K. B. Averyt, M. Tignor, and H. L. Miller, editors. 2007. Climate change 2007: the physical science basis. Contribution of Working Group I to the Fourth Assessment Report of the Intergovernmental Panel on Climate Change. Cambridge University Press, Cambridge, UK.

Stahel, C. D., S. C. Nicol, and G. J. Walker. 1986. Heat production and thermal resistance in the little penguin Eudyptula minor in relation to wind speed. Physiological and Biochemical Zoology 60:413-423.

Stearns, S. C. 1992. The evolution of life histories. Oxford University Press, Oxford, UK.

Stenseth, N. C., A. Mysterud, G. Ottersen, J. W. Hurrell, K.-S. Chan, and M. Lima. 2002. Ecological effects of climate fluctuations. Science 297:1292-1296.

Sutherland, D. R., and P. Dann. 2012. Population size estimates of burrowing seabirds robust to variation in burrow occupancy: linking surveys with demographics in little penguins. Ibis 154:488-498.

Trathan, P. N., J. Forcada, and E. J. Murphy. 2007. Environmental forcing and southern ocean marine predator populations: effects of climate change and variability. Philosophical Transactions of the Royal Society B: Biological Sciences 362:23512365.

Visser, M. E. 2008. Keeping up with a warming world: assessing the rate of adaptation to climate change. Proceedings of the Royal Society of London B: Biological Sciences 275:649-659.

Walther, G.-R. 2010. Community and ecosystem responses to recent climate change. Philosophical Transactions of the Royal Society B: Biological Sciences 365:2019-2024.

Walther, G. R., E. Post, P. Convey, A. Menzel, C. Parmesan, T. Beebee, J. M. Fromentin, O. Hoegh-Guldberg, and F. Bairlein. 2002. Ecological responses to recent climate change. Nature 416:389-395.

Weavers, B. W. 1992. Seasonal foraging ranges and travels at sea of little penguins Eudyptula minor, determined by radiotracking. Emu 91:302-317.

Weimerskirch H., Guionnet T., Martin J., Shaffer S.A., Costa D.P. 2000. Fast and fuel efficient? Optimal use of wind by flying albatrosses. Proceedings of the Royal Society of London B: Biological Sciences 267(1455),1869-1874. 
Weimerskirch, H., M. Louzao, S. de Grissac, and K. Delord. 2012. Changes in wind pattern alter albatross distribution and life-history traits. Science 335:211-214.

Williams, T. D. 1995. The penguins. Oxford University Press, Oxford, UK. 230-238.

Wu, L., et al. 2012. Enhanced warming over the global subtropical western boundary currents. Nature Climate Change 3:161-166.
Young, I. R., S. Zieger, and A. V. Babanin. 2011. Global trends in wind speed and wave height. Science 22:451-455.

Zann, R. A., S. R. Morton, K. R. Jones, and N. T. Burley. 1995. The timing of breeding by zebra finches in relation to rainfall in central Australia. Emu 95:208-222.

Zuur, A. F., E. N. Ieno, and C. S. Elphick. 2010. A protocol for data exploration to avoid common statistical problems. Methods in Ecology and Evolution 1:3-14.

\section{SUPPORTING INFORMATION}

Additional supporting information may be found in the online version of this article at http://onlinelibrary.wiley.com/ doi/10.1890/14-2124.1/suppinfo 